\title{
Un variant de protéine-prion dans une maladie génétique
}

Les prions sont les constituants principaux de particules protéiques qui s'accumulent dans le système nerveux lors d'encéphalopathies dégénératives animales (tremblante du mouton et de la chèvre) et humaines (maladie de Creutzfeldt-Jakob, kuru). Une nouvelle parue fin 1986 $\left(\mathrm{m} / \mathrm{s} n^{\circ} 10\right.$, vol.2, p. 588) faisait le point des connaissances à cette date : les prions sont synthétisés normalement sous forme de chaînes de 245 acides aminés; leur gène, qui ne compte qu'un exon, a pu être localisé [1] chez la souris sur le chromosome 2 , et chez l'homme sur le bras court du chromosome 20. Il s'agit donc d'une protéine normalement synthétisée, dont l'ARN messager est aisément détecté. La controverse vient du fait que les prions normalement ne s'accumulent pas, alors qu'ils le font dans la tremblante. Il se forme dans ce cas une protéine appelée PrPs (de scrapie, équivalent anglais de tremblante), au lieu de la PrP habituelle. La PrPs, transmise au hamster ou à la souris, en l'absence apparente d'ADN, provoque l'infection. La PrP a subi au moins des modifications post-traductionnelles, puisqu'elle est devenue résistante à la digestion par des protéases, ainsi qu'à la phospholipase $\mathrm{C}$, qui rompt la liaison glycosyl-phosphatidylinositol du prion normal à la membrane. Un argument important en faveur de l'importance des prions a été fourni, chez la souris, par la découverte de deux allèles, définis par un polymorphisme de restriction, qui gouvernent la durée d'incubation de la tremblante après infection expérimentale.
L'équipe de Prusiner (San Francisco, USA), qui compte à son actif l'essentiel des travaux sur les prions, vient de faire une découverte de grande portée. Elle concerne le syndrome de Gerstmann-Sträussler, affection exceptionnelle, voisine mais différente de la maladie de CreutzfeldtJakob. Ce syndrome semble transmis comme un caractère autosomique dominant; il est marqué, à l'âge adulte, par une ataxie ou une démence, et évolue en un à dix ans. Des plaques contenant des prions qui résistent aux protéases sont trouvées dans le cerveau des malades. Hsiao et al. [2] ont séquencé les deux allèles contenant une phase ouverte dans deux familles, une américaine et une britannique; chez les sujets atteints, une même mutation a été trouvée à l'état hétérozygote; elle consiste en un remplacement d'une cytosine par une thymine en seconde position du codon 102, facilement reconnaissable car elle crée un nouveau site pour l'enzyme de restriction DdeI. Elle provoque un changement d'une proline en une leucine. L'anomalie n'a été retrouvée ni chez les membres non atteints des deux familles, ni chez 100 témoins, ni chez 15 malades porteurs d'autres maladies à prions. Une étude de liaison dans les deux familles conduit à un lod score de 3,3 , très significatif. La notion d'une même mutation survenant indépendamment - les deux familles n'ont apparemment aucun lien de parenté - a été fortement renforcée par l'observation de deux familles japonaises non apparentées et qui la possèdent aussi (dans [3]). On est ainsi conduit à penser que cette mutation ne représente pas seulement un marqueur génétique, mais qu'elle joue un rôle causal dans la genèse du syndrome. Il sera intéressant de vérifier sur les protéines que seule celle qui est mutée s'accumule.

Quelle portée a cette découverte sur le problème général des prions? On pourrait faire l'hypothèse que la mutation rend plus aisée la formation de PrPs, qui se comporterait ensuite comme un agent infectieux transmissible. D'autre part, puisque les hétérozygotes sont atteints, une néomutation ou même une mutation somatique pourrait donner naissance à une forme sporadique de Gerstmann-Sträussler. Un autre type de mutation, non encore découvert, pourrait être en cause dans la maladie de Creutzfeldt-Jakob.

De toutes façons, l'irruption de la génétique donne une dimension nouvelle au problème de fond, à savoir si une protéine - à la suite ou non d'une mutation portant sur son gène de structure - peut être infectieuse par elle-même.

\section{J.-C.D}

1. Sparkes RS, Simon M, Cohn VH, et al. Assignment of the human and mouse prion protein genes to homologous chromosomes. Proc Natl Acad Sci USA 1986; 83 : 7358-62.

2. Hsiao K, Baker HF, Crow TJ, et al. Linkage of a prion protein missence variant to Gerstmann-Sträussler syndrome. Nature $1989 ; 338: 342-5$.

3. Weissmann C. Sheep disease in human clothing. Nature 1989; 338 : 298-9. 